\title{
ARTIGOS
}

Submetido em 01.12.2019. Aprovado em 29.07.2020

Avaliado pelo processo double blind review. Editor científico: Mateus Canniatti Ponchio

Versão traduzida | DOI: http://dx.doi.org/10.1590/So034-759020210404X

\section{DESENVOLVENDO OS COMPONENTES DA ORIENTAÇÃO PARA O MERCADO PARA MELHORAR O DESEMPENHO DE PEQUENAS E MÉDIAS EMPRESAS}

\author{
Designing architectural marketing capabilities to increase the performance of small and medium- \\ sized enterprises

\section{Diseño de capacidades de marketing arquitectónico para aumentar el rendimiento de pequeñas} \\ y medianas empresas
}

Mulyana Mulyana ${ }^{1}$ | mulyana@unissula.ac.id | ORCID: 0000-0002-7887-5195 Zainuddin Zakaria² | hzainudd@uitm.edu.my | ORCID: 0000-0003-4907-402X Rosman Mahmood² | rosmanma@uitm.edu.my | ORCID: 0000-0003-3316-1727

\author{
${ }^{1}$ Universitas Islam Sultan Agung, Department of Management Science, Faculty of Economics, Semarang, Indonésia \\ ${ }^{2}$ Universiti Teknologi MARA, Faculty of Business and Management, Dungun, Terengganu, Malásia
}

\section{RESUMO}

Este estudo tem como objetivo examinar a relação entre os componentes da orientação para o mercado - orientação para o cliente, orientação para a concorrência, coordenação interfuncional e capacidades de arquitetura de marketing - e o desempenho da empresa. Pesquisadores vêm tentando encontrar uma solução para a lacuna existente entre a orientação para o mercado e o desempenho da empresa. Os sujeitos deste estudo foram 358 proprietários ou gestores de pequenas e médias empresas (PMEs) em Java Central, na Indonésia. A pesquisa utilizou Modelagem de Equações Estruturais (SEM) para analisar os dados. Os resultados mostraram que a Orientação para o Cliente (OCL), a Orientação para Concorrência (OC) e a Coordenação Interfuncional (CI) tiveram uma influência significativa nas Capacidades de Arquitetura de Marketing (CAM) e no Desempenho da Empresa (DE), e que as CAM também tiveram uma influência significativa no desempenho da empresa. Além disso, as CAM podem mediar a relação entre a orientação para o cliente, a orientação para a concorrência, a coordenação interfuncional e 0 desempenho da empresa.

PALAVRAS-CHAVE | Orientação para o cliente, orientação para a concorrência, coordenação interfuncional, capacidades de arquitetura de marketing, desempenho da empresa.

\section{ABSTRACT}

This study aims to examine the relationship between the components of market orientation, i.e., customer orientation, competitor orientation, interfunctional coordination, architectural marketing capabilities and business performance. Researchers have been trying to find a solution for the gap that exists between market orientation and business performance. The respondents of this study were the owners or heads of 358 SMEs located in Central Java, Indonesia. This study used structural equation modeling (SEM) for data analysis. The results showed that customer orientation (CSO), competitor orientation (CO), and inter-functional coordination (IC) have a significant effect on architectural marketing capabilities (AMC) and business performance (BP), and that AMC also has a significant effect on business performance. Furthermore, architectural marketing capabilities can mediate the relationship between customer orientation, competitor orientation, inter-functional coordination and business performance.

KEYWORDS / Customer orientation, competitor orientation, inter-functional coordination, architectural marketing capabilities, business performance

\section{RESUMEN}

Este estudio tiene como objetivo determinar el efecto de los componentes de orientación del mercado, a saber, la orientación al cliente, la orientación a la competencia, la coordinación entre funciones y recursos de marketing arquitectónico en el rendimiento del negocio. Los investigadores están tratando de encontrar una solución para la brecha que existe entre la orientación al mercado y el desempeño comercial. Los encuestados de este estudio fueron 358 propietarios o líderes de pymes en Java Central, Indonesia. Esta investigación utilizó el modelado de ecuaciones estructurales (SEM) como análisis de datos. Los resultados mostraron que la orientación al cliente (CSO), la orientación a la competencia (CO) y la coordinación interfuncional (IC) tuvieron una influencia significativa en la capacidad de marketing arquitectónico (AMC) y en el desempeño comercial (BP). Asimismo, la AMC tuvo una influencia significativa en el desempeño comercial. Además, las capacidades de marketing arquitectónico pueden mediar la relación entre la orientación al cliente, la orientación a la competencia, la coordinación interfuncional y el rendimiento empresarial.

PALABRAS CLAVE I Orientación al cliente, orientación a la competencia, coordinación funcional, habilidades de marketing arquitectónico, desempeño comercial. 


\section{INTRODUÇÃO}

Já foram realizadas pesquisas sobre a relação entre a orientação para o mercado e o desempenho da empresa. A orientação para o mercado foi inicialmente introduzida como um componente que afeta o desempenho da empresa (Dubihlela \& Sandada, 2014; Kaswuri, Ramayah, \& Aldakhil, 2017). Espera-se que o estudo da relação entre os componentes da orientação para o mercado (orientação para o cliente, orientação para a concorrência e coordenação interfuncional) e o desempenho da empresa forneça resultados abrangentes no campo da gestão de empresas. Os resultados de estudos anteriores mostram que a orientação para o mercado é a chave para o sucesso no que diz respeito ao desempenho da empresa (Udriyah, Tham, \& Azam, 2019). Embora alguns estudos expliquem que a orientação para o mercado contribui para a melhoria do desempenho da empresa (Herman, Hady, \& Arafah, 2018; Hwang \& Chung, 2018; Mamun, Mohiuddin, Fazal, \& Ahmad, 2018), outros estudos mostram resultados diferentes: que não há relação entre a orientação para o mercado e o desempenho da empresa (Gholami \& Birjandi, 2016; Idrus, Ahmar, \& Abdussakir, 2018). A orientação para o mercado também não é capaz de melhorar o desempenho financeiro (Ho, Nguyen, Adhikari, Miles, \& Bonney, 2018), e empresas que são sempre proativas na orientação para o mercado têm sido incapazes de alcançar um desempenho superior (Kharabsheh, Jarrar, \& Simenonva, 2014).

A orientação para o cliente, a orientação para a concorrência e a coordenação interfuncional são componentes da orientação para o mercado, que é um conjunto de comportamentos culturais organizacionais (Narver \& Slater, 1990). Espera-se que a orientação para o cliente, como comportamento da cultura organizacional, seja capaz de compreender as necessidades e demandas dos clientes. A orientação para a concorrência deve compreender os pontos fortes e fracos e as estratégias dos concorrentes, enquanto a coordenação interfuncional deve construir relacionamentos harmoniosos entre as funções, de modo a aproveitar as oportunidades de mercado. Espera-se que a compreensão dos componentes da orientação para o mercado facilite o planejamento e a implementação do marketing.

0 desenvolvimento das capacidades de arquitetura de marketing é um processo que inclui o planejamento e a implementação de estratégias de marketing (Morgan, 2012). 0 marketing precisa ser planejado para definir metas de mercado e desenvolver estratégias de marketing eficazes, ao passo que a implementação de um processo de marketing é necessária para facilitar a alocação de recursos de marketing, interpretar as estratégias de marketing de maneira adequada e introduzir programas de marketing de modo eficaz (Vorhies \& Morgan, 2005). Poucas pesquisas têm sido realizadas sobre o efeito da orientação para o mercado nas capacidades de arquitetura de marketing. Às vezes, a orientação para o mercado e as capacidades de arquitetura de marketing são tratadas como variáveis independentes que afetam o desempenho da empresa (Morgan, Slotegraaf, \& Vorhies, 2009). Da mesma forma, a compreensão da relação entre o desempenho da empresa e a orientação para o mercado será mais completa se os objetivos forem compreendidos, de modo que o papel do mediador determinará a força da relação (Han, Kim, Han, Kim, \& Srivastava, 1998).

As divergências nos resultados das pesquisas acima referidas podem ser resultantes de medições inadequadas, do posicionamento inadequado de mediadores ou devido às várias técnicas de coleta ou análise de dados utilizadas. Devido a essa lacuna nas pesquisas, ainda há oportunidades para a realização de mais pesquisas, e este estudo visa preencher a lacuna entre os componentes da orientação para o mercado e o desempenho da empresa. Isso será feito tomando-se as capacidades de arquitetura de marketing como variável mediadora. Uma compreensão detalhada da relação entre as capacidades de arquitetura de marketing, os componentes da orientação para o mercado e o desempenho da empresa ainda é necessária para a ampliação dos conhecimentos disponíveis, especialmente no campo da gestão de marketing. 


\section{ARCABOUÇO TEÓRICO}

\section{0 desenvolvimento conceitual das capacidades de arquitetura de marketing}

A visão baseada em recursos (VBR) afirma que as capacidades são um recurso que, se bem gerenciado, constituirá uma vantagem para a empresa (Barney, 1991). As capacidades de uma empresa consistem em sua condição de ser capaz de explorar os recursos de que dispõe (Javidan, 1998). As capacidades podem ser desenvolvidas se os indivíduos e grupos forem capazes de explorar seus conhecimentos e habilidades para apoiar a realização dos objetivos da empresa (Morgan, 2012).

As capacidades de arquitetura referem-se ao processo de seleção, integração e gerenciamento de habilidades especiais e compartilhamento de recursos entre funções em uma organização (Galunic \& Rodan, 1998; Henderson \& Clark, 1990). A arquitetura do marketing inclui o processo de definição de objetivos de marketing, formulação de estratégias de marketing (Slotegraaf \& Dickson, 2004; Vorhies \& Morgan, 2003) e implementação de decisões de estratégia de marketing apoiadas pelos recursos da empresa (Capron \& Hulland, 1999; Vorhies \& Morgan, 2005).

Capacidades de marketing são o processo integrativo criado para aplicar conhecimentos, habilidades e recursos da empresa de modo a atender as necessidades dos mercados-alvo (Day, 1994; Hamdani, Susanto, \& Maulani, 2018). Neste estudo, as capacidades de arquitetura de marketing são a capacidade de gerenciar recursos para o planejamento e implementação de planos de marketing a fim de atingir os mercados-alvo, conforme medido pelas capacidades empregadas no planejamento de mercado, desenvolvimento de estratégias de marketing criativas, precisão na escolha de mercados-alvo, alocação eficaz de recursos de marketing, interpretação de estratégias de marketing, bem como sua transformação em ação e sua execução de maneira adequada.

\section{Orientação para o mercado}

De acordo com Naver e Slater (1990), o estudo dos componentes da orientação para o mercado, que incluem orientação para o cliente, orientação para concorrência e coordenação interfuncional, faz parte da orientação organizacional para o mercado. A orientação para o cliente deve ser capaz de entender suficientemente os compradores-alvo ao longo do tempo, de modo a criar valor para eles. A orientação para a concorrência deve obter informações sobre os concorrentes principais e potenciais, bem como compreender os pontos fortes e fracos dos concorrentes de curto e longo prazos. A coordenação interfuncional é útil para a coordenação de recursos com vistas a criar valor superior para o mercado-alvo. Os estudos sobre orientação para o mercado no contexto do marketing reconhecem o papel da cultura corporativa em influenciar a orientação para o mercado. Eles também introduzem outras perspectivas que são úteis para teste de teoria, benchmarking e rastreamento (Jaworski \& Kohli, 1993). A orientação para o mercado envolve clientes e concorrentes, reconhece o papel da cultura organizacional no apoio à orientação para o mercado e reconhece a orientação para o mercado como a base da estratégia corporativa. As empresas orientadas para o mercado podem desenvolver e disseminar capacidades de marketing específicas e distintas para se diferenciarem das empresas concorrentes. Pesquisa de mercado, precificação, desenvolvimento de produtos, canais de distribuição, promoção e gerenciamento de mercado são necessários para fortalecer as capacidades de marketing da empresa (Vorhies, Harker, \& Rao, 1999). A orientação para o mercado pode melhorar as capacidades de implementação do marketing, o que, por sua vez, fortalece o desempenho comercial com foco nos clientes, isto é, em atraí-los e retê-los. 


\section{Desempenho da empresa}

De acordo com Rahman et al. (2018), o método utilizado para medir o desempenho da empresa é multidimensional em relação ao tema desejado. Alguns pesquisadores utilizam a dimensão do crescimento como proxy para o desempenho da empresa (Cho \& Pucik, 2005; Morgan et al., 2009). De acordo com Stam, Arzlanian e Elfring (2014), o desempenho empresarial em pequenas empresas pode ser explicado em termos de três dimensões: desempenho de crescimento, lucratividade e desempenho não financeiro. Lee, Kim, Seo e Hight (2015) definem o desempenho empresarial como as realizações da empresa, incluindo seu desempenho financeiro e não financeiro. $O$ desempenho financeiro é medido pelas percepções dos sujeitos pesquisados com relação ao atingimento de seus objetivos de lucro líquido e vendas, aumento da receita líquida e vendas, e atingimento do número planejado de contratos. 0 desempenho não financeiro é medido por indicadores das percepções dos sujeitos pesquisados sobre a melhoria das capacidades de novos produtos e serviços, e aumento da satisfação dos funcionários e clientes. Devido à má governança nas PMEs, as percepções dos sujeitos pesquisados são utilizadas como abordagem para medir o desempenho da empresa. Tal desempenho resulta das atividades operacionais da organização, as quais incluem resultados internos e externos medidos pelo ROA, crescimento das vendas, crescimento da participação no mercado e crescimento do lucro (Nuryakin, Didiek, \& Budi, 2018). Neste estudo, o desempenho empresarial é a capacidade da empresa de atingir seus objetivos financeiros e de marketing, conforme medido por indicadores de crescimento nos resultados das vendas, aumento do número de clientes, expansão das áreas de vendas, crescimento dos lucros e crescimento do capital da empresa.

\section{ESTRUTURA CONCEITUAL E HIPÓTESES DE PESQUISA}

\section{Orientação para o cliente e capacidades de arquitetura de marketing}

A orientação para o cliente é a capacidade da empresa de observar e avaliar tendências nas necessidades de seus clientes, o que a incentiva a inovar em termos de seus produtos e serviços, a fim de satisfazer esses clientes (Micheels \& Gow, 2008; Sadikoglu \& Zehir, 2010). O conhecimento do mercado é muito útil para orientar o planejamento de marketing que esteja em harmonia com as mudanças ambientais (Moorman \& Slotegraaf, 1999). Os recursos de uma empresa serão utilizados para desenvolver uma estratégia de marketing (Davcik \& Sharma, 2016), que inclui conhecimentos, capacidade financeira, recursos humanos, networking, informação e desenvolvimento (Hamdani et al., 2018). Outros estudos mostram que os recursos de marketing na forma de conhecimentos, informação e recursos humanos podem ser utilizados como base para o desenvolvimento de estratégias de marketing (Foumani \& Chirani, 2012). As empresas que entendem as necessidades de seus clientes, criam valor agregado e fornecem serviços pós-venda estarão mais bem equipadas para reorganizar seus recursos de marketing. Portanto, a capacidade dos recursos de marketing de entender os clientes completamente permite que as empresas melhorem sua capacidade de planejar e implementar suas iniciativas de marketing.

H1: Há uma relação positiva e significativa entre OCL e CAM.

\section{Orientação para a concorrência e capacidades de arquitetura de marketing}

Uma orientação para a concorrência permite que as organizações determinem, avaliem e respondam aos pontos fortes e fracos dos concorrentes (Naver \& Slater, 1990). As empresas orientadas para a concorrência rastreiam 
as ações de seus concorrentes e emulam seus produtos por meio da criação de inovações adicionais em seus próprios produtos e serviços (Lukas \& Ferrell, 2000). Uma cultura de orientação para o mercado afetará as capacidades de marketing de uma empresa, especialmente o planejamento e implementação de seu mix de marketing (O'Cass, Ngo, \& Siahtiri, 2012). As capacidades de arquitetura de marketing podem ser desenvolvidas por meio de um planejamento de marketing abrangente com base em análises ambientais e informações sobre a concorrência (Hamdani et al., 2018). As empresas que entendem os pontos fortes e fracos de seus concorrentes e rastreiam suas ações e estratégias desde o início estarão mais bem preparadas para desenvolver suas próprias atividades de marketing. Portanto, a capacidade de uma empresa de compreender os pontos fortes e fracos de seus concorrentes e rastrear suas estratégias permite melhorar sua própria capacidade de planejar e implementar suas iniciativas de marketing.

H2: Há uma relação positiva e significativa entre OC e CAM.

\section{Coordenação interfuncional e capacidades de arquitetura de marketing}

A coordenação interfuncional mostra o nível de envolvimento de todas as funções internas da organização para compartilhar informações e construir relações harmoniosas de modo a atingir seu mercado-alvo (Song et al., 2000; Mohsen, 2013). A coordenação interfuncional é necessária para compartilhar informações a fim de encontrar ideias que sejam criativas (Wang \& Miao, 2015). Ao utilizar recursos de marketing baseados em capacidades e conhecimentos e que se concentram principalmente em decisões e estratégias de implementação de marketing, as capacidades de arquitetura de marketing formam a base do modelo utilizado para desenvolver um marketing orientado para resultados (Hamdani et al., 2018). As capacidades de marketing também incluem habilidades coletivas, o acúmulo de conhecimentos e a capacidade de coordenar as atividades de marketing e desenvolvê-las para que se tornem ativos da empresa. A capacidade de planejar o marketing está relacionada à capacidade de desenvolver estratégias de marketing adequadas, o que é alcançado por meio do alinhamento de recursos às condições de mercado, facilitando o atingimento dos objetivos da empresa (Mokwa, McKee, Varadarajan, \& Conant, 1992). Empresas que alcançam uma coordenação interfuncional harmoniosa facilitarão o compartilhamento de informações e recursos para aproveitar as oportunidades de mercado. Assim, a coordenação interfuncional harmoniosa permite que as empresas melhorem sua capacidade de planejar e implementar suas iniciativas de marketing.

H3: Há uma relação positiva e significativa entre Cl e CAM.

\section{Orientação para o cliente e desempenho da empresa}

Segundo Naver e Slater (1990), a orientação para o cliente, a orientação para a concorrência e a coordenação interfuncional, como componentes da orientação para o mercado, podem ser utilizadas para criar valor superior para a empresa. 0 objetivo da orientação para o mercado é fornecer valor agregado aos clientes com base em seus inputs e na análise da concorrência (Avlonitis \& Giannopoulos, 2012). A orientação para o cliente, em particular, desempenha um grande papel no apoio a melhorias no desempenho da empresa (Asomaning \& Abdulai, 2015; Feng, Wang, Lawton, \& Luo, 2019; Neneh, 2018; Ogbonna \& Ogwo, 2013; Suliyanto \& Rahab, 2012) e no desempenho financeiro (Taofik, Halim, \& Ibrahim, 2018). A capacidade de entender as necessidades e demandas dos clientes e criar para eles o melhor valor tornará a empresa mais bem equipada para lidar com as mudanças 
ambientais. Portanto, a capacidade da empresa de atender às expectativas do cliente tem potencial para aumentar as vendas e lucros e o crescimento do capital e do número de clientes.

$$
\text { H4: Há uma relação positiva e significativa entre OCL e DE. }
$$

\section{Orientação para a concorrência e desempenho da empresa}

As informações sobre o mercado são utilizadas pelas empresas como um guia para o planejamento do marketing de modo a se adequar às mudanças ambientais (Day, 1994). 0 desempenho de uma empresa é fortemente influenciado pela orientação para a concorrência, pela capacidade da empresa de compreender os pontos fortes e fracos dos concorrentes e suas estratégias e táticas (Chin, Lo, \& Ramayah, 2013; Suliyanto \& Rahab, 2012; Wang, Chen, \& Chen, 2012). A velocidade de resposta às ações dos concorrentes e de oferta de produtos novos e melhores tem potencial para aumentar as vendas e os lucros, de modo que a orientação para a concorrência pode ser um determinante do desempenho financeiro (Taofik et al., 2018). As empresas que compreendem os pontos fortes e fracos de seus concorrentes e conhecem suas estratégias e ações serão capazes de responder rapidamente. Portanto, a rapidez em compreender e responder às estratégias dos concorrentes permite que as empresas formulem novas políticas de uma maneira melhor e, potencialmente, aprimorem seu desempenho.

H5: Há uma relação positiva e significativa entre OC e DE.

\section{Coordenação interfuncional e desempenho da empresa}

De acordo com Kanovska e Tomaskova (2012), a coordenação interfuncional é um processo pelo qual todas as funções internas são harmonizadas. Assim, as organizações que desejam vencer os concorrentes devem ser capazes de integrar suas funções de maneira otimizada. A coordenação interfuncional é um componente que pode apoiar a sobrevivência organizacional (Ajay \& Jaworski, 1990). Esse componente de coordenação em uma organização inclui o nível de envolvimento, a qualidade das informações e as relações harmoniosas entre as funções (Mohsen, 2013). Namusonge e Nuriri (2017) mostraram que a coordenação interfuncional tem uma forte relação com o desempenho da empresa, podendo produzir novos líderes de produto que melhorarão esse desempenho. A coordenação interfuncional harmoniosa facilita que uma empresa compartilhe recursos e informações a fim de aproveitar as oportunidades de mercado por ela almejadas. Portanto, a coordenação interfuncional utilizada para atingir o mercado-alvo tem o potencial de melhorar o desempenho da empresa.

H6: Existe uma relação positiva e significativa entre $\mathrm{Cl}$ e DE.

\section{Capacidades de arquitetura de mercado e desempenho da empresa}

Empírica e teoricamente, as capacidades de marketing podem melhorar o desempenho de uma empresa (Morgan, 2012), enquanto o desenvolvimento de capacidades de arquitetura de marketing pode melhorar diretamente 0 desempenho de uma empresa (Hamdani et al., 2018; Seifi \& Dalvi, 2014). As capacidades de arquitetura de marketing são necessárias para identificar a força dos recursos disponíveis, para que a empresa possa formular um planejamento estratégico para a implementação de iniciativas de marketing adequadas que terão um impacto na melhoria do desempenho da empresa e levarão ao crescimento sustentável (Hamdani et al., 2018). Da mesma forma, as capacidades de arquitetura organizacional podem determinar o sucesso de uma empresa (Chikwe, Biriowu, \& Harcourt, 2018). As capacidades de arquitetura de marketing também são necessárias para identificar a 
força dos recursos de uma empresa para que ela possa preparar seus planos de marketing e implementá-los de maneira adequada. A capacidade de explorar o potencial dos recursos de marketing de uma empresa fornecerá suporte quando ela estiver desenvolvendo suas iniciativas de marketing. Portanto, o conjunto de habilidades organizadas para tirar proveito dos recursos de marketing de uma empresa terá o potencial de melhorar o seu desempenho.

H7: Há uma relação positiva e significativa entre CAM e DE.

O conceito de desenvolvimento de um modelo para testar hipóteses é mostrado na Figura 1.

Figura 1. Estrutura conceitual

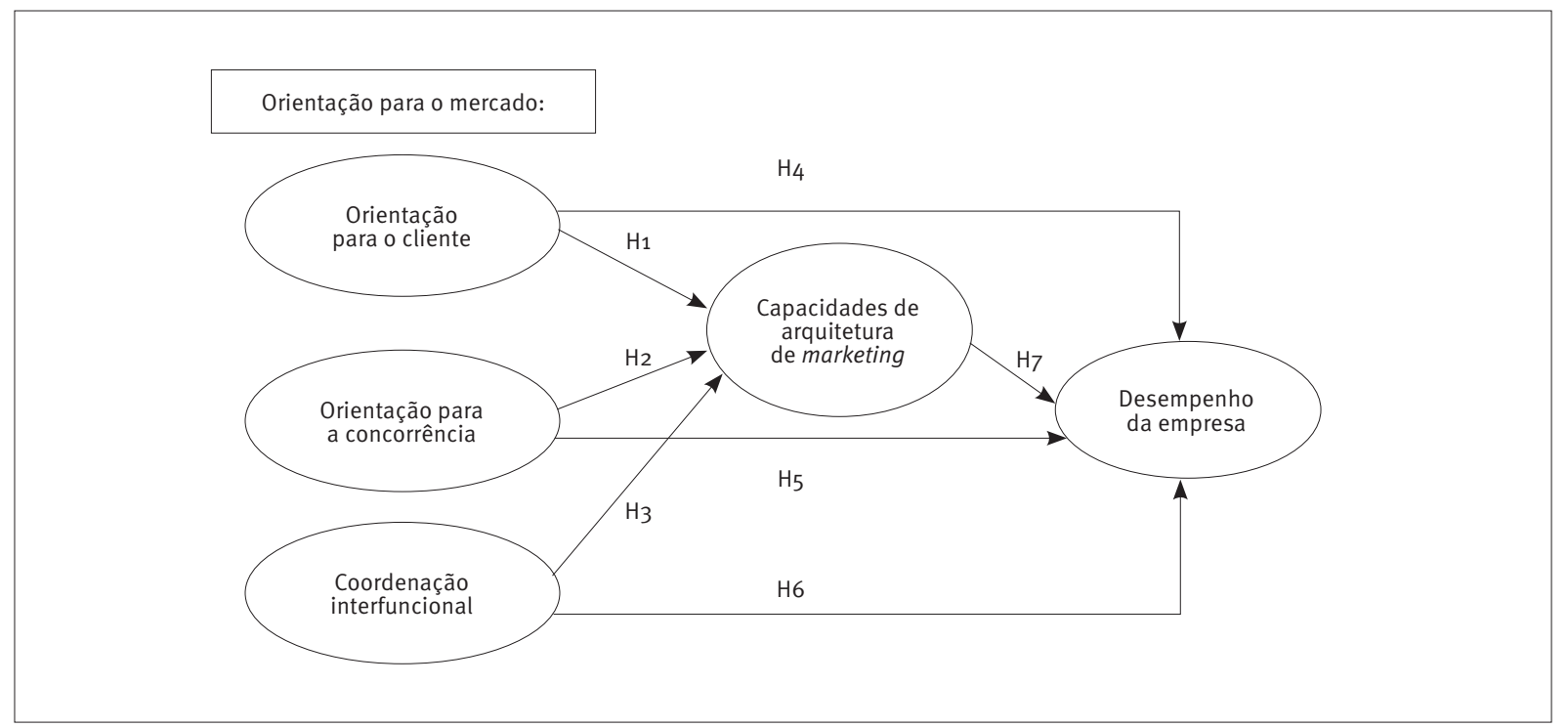

\section{MÉTODO DE PESQUISA}

\section{Coleta de dados e a amostra}

Esta pesquisa foi conduzida com pequenas e médias empresas localizadas em Java Central, Indonésia. Os dados foram extraídos de questionários e entrevistas, para garantir a precisão das respostas dos sujeitos pesquisados. Os questionários foram distribuídos a 483 sujeitos em 12 cidades/distritos na Província de Java Central. Java Central foi selecionada como amostra devido à sua importância para o desenvolvimento econômico da Indonésia. Após a seleção e validação, 358 sujeitos atendiam aos requisitos da pesquisa. A amostra é composta por $67,3 \%$ de mulheres e 32,7\% de homens com idades entre 20 e 65 anos, que possuem entre 4 e 41 anos de experiência empresarial. Dos setores de negócios envolvidos, 45,5\% estão em moda, 22,4\%, em calçados, 19,6\%, em bolsas e 12,5\%, em acessórios. Para testar os efeitos potenciais do método comum, o teste de fator único de Harman foi realizado utilizando um programa SPSS. Os resultados do teste mostram que o fator único pode explicar 33,4\% da variância total e está abaixo do valor de corte de 50\% (Podsakoff, Mackenzie, \& Podsakoff, 2012). Portanto, esse valor atende aos requisitos especificados no teste do viés do método comum. 


\section{Medições}

Este estudo utilizou dados primários coletados pelos pesquisadores por meio de uma pesquisa. O questionário foi concebido para medir as respostas dos participantes a todos os construtos e utilizou uma escala de avaliação de 1 a 10 (Hair, Black, Babin, \& Anderson, 2010). O nível de pontuação permitia que os participantes escolhessem desde a pontuação mais baixa (1) até a pontuação mais alta (10). Todos os construtos foram medidos utilizando uma pontuação de 1 para "Discordo totalmente" a 10 para "Concordo totalmente". A escala é utilizada para medir as variáveis de pesquisa, que incluem as capacidades de arquitetura de marketing, a orientação para o cliente, a orientação para a concorrência, a coordenação interfuncional e o desempenho da empresa. A medição dos construtos neste estudo foi realizada a partir da literatura disponível, sendo algumas modificações realizadas para se adequar ao contexto da pesquisa. A orientação para o cliente é uma adaptação a partir de Asikhia (2014) e Suleiman e Agti (2011), que realizaram a medição utilizando os indicadores de satisfação do consumidor, compreensão das necessidades do consumidor, criação de produtos de valor agregado para os consumidores e prestação de serviço pós-venda. A orientação para a concorrência, que foi adaptada a partir de Asikhia (2014) e Suleiman e Agti (2011), é medida utilizando indicadores de resposta rápida às ações dos concorrentes, criação de produtos superiores, resposta rápida às mudanças nos preços dos concorrentes e monitoramento dos esforços de marketing dos concorrentes. A coordenação interfuncional foi adaptada a partir de Asikhia (2014) e Suleiman e Agti (2011), que realizaram sua medição utilizando indicadores de trabalho em equipe no atendimento de clientes, compartilhamento de informações, cooperação para respostas às ações dos concorrentes, cooperação para a formulação de estratégias e cooperação para criação de produtos superiores. As capacidades de arquitetura de marketing foram adaptadas a partir de Trez e Luce (2012) e Vorhies e Morgan, (2005), que realizaram sua medição utilizando indicadores de habilidades em planejamento de mercado, desenvolvimento de estratégias de marketing criativas, precisão na escolha dos mercados-alvo, alocação eficaz de recursos de marketing, transformação de estratégias de marketing em ações e busca adequada de uma estratégia de marketing. 0 desempenho da empresa foi adaptado a partir da visão de Morgan (2012) e Nuryakin et al. (2018), que realizaram sua medição por meio de cinco indicadores: crescimento das vendas, crescimento do número de clientes, expansão da área de vendas, aumento dos lucros e crescimento do capital da empresa.

\section{Análise de dados e modelos de medição}

A abordagem da modelagem de equações estruturais (SEM) será utilizada para processar os dados coletados e facilitar a testagem das hipóteses. 0 objetivo deste estudo é examinar a relação entre a orientação para o cliente, a orientação para a concorrência, a coordenação interfuncional, as capacidades de arquitetura de marketing e o desempenho da empresa. 0 índice de bondade de ajuste foi utilizado para avaliar o ajuste do SEM recomendado, incluindo: estatística de qui-quadrado; índice de ajuste normal (NFI); índice de ajuste comparado (CFI); índice de Tucker-Lewis (TLI); e raiz quadrada da média do erro de aproximação (RMSEA).

A Tabela 1 mostra que todos os indicadores são válidos, o que se comprova pelo valor do fator de carga, > 0,5 e p < 0,05. A Tabela 2 mostra que todos os indicadores das variáveis latentes medidas apresentam consistência interna, ou seja, todos os construtos são confiáveis (Hair et al., 2010). Os valores de confiabilidade composta (CR) > o,6 e variância extraída (VE) > 0,5 estão de acordo com os padrões de consistência interna para os indicadores medidos. As variáveis de orientação para o cliente, orientação para a concorrência, coordenação interfuncional, capacidades de arquitetura de marketing e desempenho da empresa apresentam valores de CR> 0,7 e AVE> 0,5. 
Tabela 1. Resultados da análise fatorial confirmatória para os modelos de medição

\begin{tabular}{|c|c|}
\hline Variável e indicador & Fator de carga \\
\hline \multicolumn{2}{|l|}{ Orientação para o cliente } \\
\hline Nossa estratégia de negócios é direcionada para alcançar a satisfação do cliente. & 0,696 \\
\hline Nós nos esforçamos para obter uma vantagem competitiva com base na compreensão das necessidades do cliente. & 0,721 \\
\hline Desenvolvemos estratégias para criar produtos de valor agregado para os clientes. & 0,722 \\
\hline Atribuímos grande importância ao serviço pós-venda. & 0,705 \\
\hline \multicolumn{2}{|l|}{ Orientação para a concorrência } \\
\hline Respondemos rapidamente às ações dos concorrentes que ameaçam o desenvolvimento da empresa. & 0,69 \\
\hline Criamos produtos que são melhores do que os produtos dos concorrentes. & 0,752 \\
\hline Respondemos rapidamente às mudanças nos preços dos principais concorrentes do setor. & 0,677 \\
\hline Monitoramos regularmente os esforços de marketing dos principais concorrentes. & 0,703 \\
\hline \multicolumn{2}{|l|}{ Coordenação interfuncional } \\
\hline Nós e os funcionários trabalhamos juntos em equipes para atender os clientes. & 0,684 \\
\hline Nós e os funcionários compartilhamos informações sobre os pontos fortes e fracos dos concorrentes. & 0,799 \\
\hline Nós e os funcionários trabalhamos juntos para responder às ações dos concorrentes rapidamente. & 0,724 \\
\hline Nós e os funcionários trabalhamos juntos para formular uma estratégia para lidar com os concorrentes. & 0,676 \\
\hline Todos os funcionários trabalham juntos para criar produtos superiores de acordo com os desejos dos clientes. & 0,68 \\
\hline \multicolumn{2}{|l|}{ Capacidade de arquitetura de marketing } \\
\hline Nossas habilidades no desenvolvimento de planejamento de marketing continuam a melhorar. & 0,753 \\
\hline Nossa capacidade de desenvolver estratégias de marketing criativas continua a aumentar. & 0,675 \\
\hline Nossa capacidade de segmentar e atingir mercados continua a aumentar. & 0,657 \\
\hline Nossa capacidade de alocar recursos de marketing eficazes continua a aumentar. & 0,796 \\
\hline Nossa capacidade de interpretar estratégias de marketing em ações continua a aumentar. & 0,744 \\
\hline Nossa capacidade de executar estratégias de marketing rapidamente continua a aumentar. & 0,736 \\
\hline \multicolumn{2}{|l|}{ Desempenho da empresa } \\
\hline As vendas de nossa empresa continuaram a aumentar nos últimos três anos. & 0,764 \\
\hline O número de nossos clientes continuou a aumentar nos últimos três anos. & 0,741 \\
\hline O território de vendas da nossa empresa continuou a aumentar nos últimos três anos. & 0,716 \\
\hline Os lucros de nossa empresa continuaram a aumentar nos últimos três anos. & 0,675 \\
\hline O capital da nossa empresa continuou a aumentar nos últimos três anos. & 0,741 \\
\hline
\end{tabular}


Tabela 2. Confiabilidades dos construtos, correlações e AVE

\begin{tabular}{|c|c|c|c|c|c|}
\hline$N=358$ & 1 & 2 & 3 & 4 & 5 \\
\hline Orientação para o cliente (OCL) & 0,808 & & & & \\
\hline Orientação para a concorrência (OC) & 0,438 & 0,797 & & & \\
\hline Coordenação interfuncional (Cl) & 0,546 & 0,532 & 0,871 & & \\
\hline Capacidade de arquitetura de marketing (CAM) & 0,519 & 0,499 & 0,500 & 0,871 & \\
\hline Desempenho da empresa (DE) & 0,523 & 0,513 & 0,525 & 0,552 & 0,858 \\
\hline Variância média extraída (AVE) & 0,679 & 0,797 & 0,871 & 0,781 & 0,770 \\
\hline
\end{tabular}

\section{RESULTADO E ANÁLISE}

O teste do modelo utilizando a abordagem de SEM requer que sejam atendidos vários critérios, ou seja, que o índice de bondade de ajuste esteja de acordo com os critérios recomendados pelo SEM. 0 valor do qui-quadrado = 271,016, e não é significativo em a: 0,05; AGFI: 0,928; índice GFI: 0,942; TLI: 0,991; CFI: 0,992; NFI: 928. Todos os valores atendem aos requisitos da SEM recomendada, que é $\geq 0,90$. Valor da RMSEA: 0,018, menor do que 0,08; CMIN/ DF: 1,115 , menor do que 2,00. Assim, os resultados do teste do índice de bondade de ajuste estão de acordo com a SEM recomendada. Portanto, o modelo tem bom ajuste, sendo viável para testar as relações entre os construtos.

Tabela 3. Testes das hipóteses

\begin{tabular}{|c|c|c|c|c|}
\hline Especificações das relações & $\beta$ padr. & $\beta$ não padr. & SE & C. R. \\
\hline $\mathrm{H}_{1}: \mathrm{OCL} \rightarrow \mathrm{CAM}$ & 0,202 & 0,245 & 0,094 & $2.611^{*}$ \\
\hline $\mathrm{H}_{2}: \mathrm{OC}->\mathrm{CAM}$ & 0,293 & 0,311 & 0,078 & $4.004^{\star}$ \\
\hline $\mathrm{H}_{3}: \mathrm{Cl}$-> CAM & 0,265 & 0,269 & 0,069 & $3.893^{*}$ \\
\hline $\mathrm{H}_{4}: \mathrm{OCL}>\mathrm{DE}$ & 0,181 & 0,226 & 0,095 & $2.385^{*}$ \\
\hline $\mathrm{H}_{5}: \mathrm{OC} \rightarrow \mathrm{DE}$ & 0,203 & 0,223 & 0,081 & $2.738^{\star}$ \\
\hline $\mathrm{H} 6: \mathrm{Cl}$-> DE & 0,202 & 0,212 & 0,073 & $2.920^{*}$ \\
\hline $\mathrm{H}_{7}: \mathrm{CAM}$-> DE & 0,255 & 0,263 & 0,071 & $3.688^{*}$ \\
\hline
\end{tabular}

Nota: ${ }^{*} p<0,01$

A Figura 1 e a Tabela 3 mostram que existe uma relação significativa entre OCL e CAM $(\beta$ padr. =0,202, CR $=$ 2.611, $\mathrm{p}<0,01)$, portanto a hipótese $\mathrm{H}_{1}$ é aceita. Além disso, há uma relação significativa entre OC e CAM ( $\beta$ padr. $=0,293, C R=4.004, p<0,01), C l$ e CAM ( $\beta$ padr. = 0,265, CR = 3.893, p <0,01), portanto H2 e H3 são aceitas. Há uma relação significativa entre OCL e DE ( $\beta$ padr. $=0,181, C R=2.385, p<0,01), O C$ e DE $(\beta$ padr. $=.203, C R=2.738$, $p<0,01), \mathrm{Cl}$ e DE ( $\beta$ padr. = 0,202, $\mathrm{CR}=2,90, \mathrm{p}<0,01)$, portanto $\mathrm{H}_{4}, \mathrm{H}_{5}, \mathrm{H} 6$ são aceitas. Também há uma relação significativa entre CAM e DE ( $\beta$ padr. $=0,255, C R=3.688, p<0,01)$, o que significa que H7 é aceita.Como variável interveniente, $C A M$ atende aos critérios de que $\mathrm{Cl}$, $O C$ e $O C L$ têm um efeito significativo sobre $D E$, e também que $\mathrm{Cl}, O C$ e OCL têm um efeito significativo sobre CAM, então $\mathrm{Cl}, \mathrm{OC}$ e OCL têm um efeito significativo sobre $D E$, controlando Cl, OC e OCL (Baron \& Kenny, 1986). Os resultados deste estudo mostram que há uma influência significativa e decrescente de $\mathrm{Cl}, \mathrm{OC}$ e OCL sobre DE quando a variável CAM é incluída, portanto há mediação parcial. 
A fim de testar se CAM se torna uma variável mediadora, este estudo utilizou a abordagem do teste de Sobel. A partir dos resultados do teste, demonstra-se que CAM é uma variável mediadora entre OCL e DE; o efeito de OCL sobre CAM tem $\beta$ não padr. = 0,245, $\mathrm{SE}=0,094$, e CAM sobre $D E$ tem $\beta$ não padr. $=0,263, \mathrm{SE}=0,071$. Os resultados da estatística do teste de Sobel são 2,131 e $p<0,05$, o que significa que CAM atua como variável mediadora entre OCL e DE. Os resultados dos testes também mostram que CAM se torna uma variável mediadora entre OC e DE. 0 efeito de OC sobre CAM tem $\beta$ não padr. = 0,311, SE =0,078, e CAM sobre DE tem $\beta$ não padr. =0,263, SE =0,071. As estatísticas do resultado do teste Sobel são 2,713 e p <0,05. Assim, CAM atua como variável mediadora entre OC e DE. Os resultados do teste mostram que CAM se torna uma variável mediadora entre $\mathrm{Cl}$ e $\mathrm{DE}$. $\mathrm{O}$ efeito de $\mathrm{Cl}$ sobre CAM tem $\beta$ não padr. $=0,269$, SE $=0,069$ e CAM sobre $D E$ tem $\beta$ não padr. $=0,263$, SE $=0,071$. Os resultados da estatística do teste de Sobel são 2,685 e p <0,05, o que significa que CAM atua como variável mediadora entre Cl e DE.

\section{Figura 2. Modelo estrutural completo}

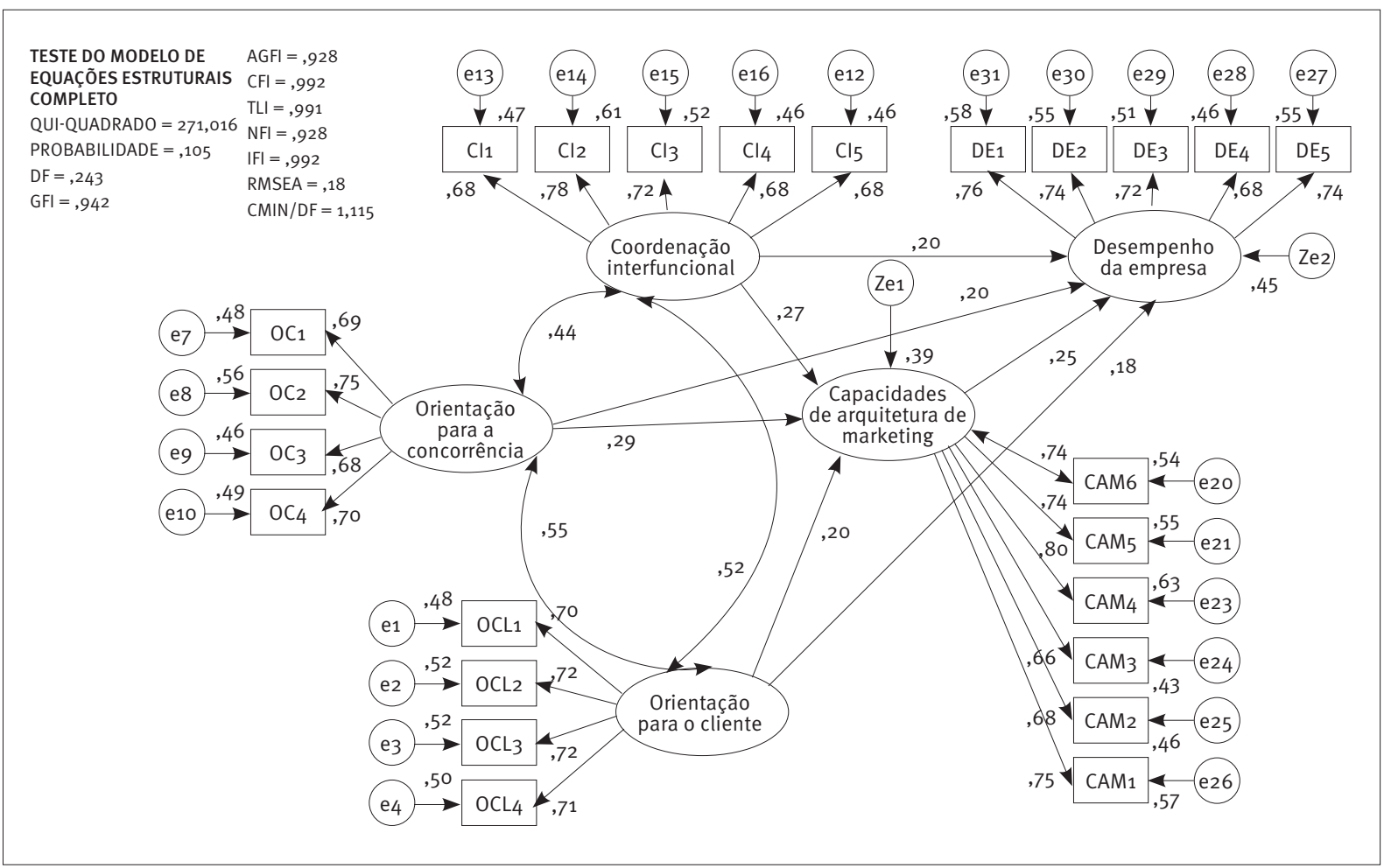

\section{DISCUSSÃO}

A orientação para o cliente possui uma relação significativa com as capacidades de arquitetura de marketing. A capacidade de atender e satisfazer as necessidades dos clientes facilita o planejamento de marketing e os programas de implementação de marketing. Os resultados deste estudo estão em consonância com os achados que afirmam que o conhecimento de informações de mercado pode orientar as empresas para que realizem um planejamento de marketing que esteja em harmonia com as mudanças ambientais (Moorman \& Slotegraaf, 1999). 0 sucesso de produtos de marketing alinhados às expectativas dos clientes é determinado pelas estratégias de marketing e pela capacidade de implementá-las (Day \& Wensley, 1988). As PMEs que entendem as necessidades e 
desejos de seus clientes terão facilidade para realizar um planejamento e implementar programas de marketing para a satisfação dos clientes.

A orientação para a concorrência apresenta uma relação significativa com as capacidades de arquitetura de marketing. A capacidade de compreender os pontos fortes e fracos dos concorrentes e suas estratégias guia o planejamento de marketing e as estratégias de implementação de marketing. Os resultados deste estudo estão em consonância com as conclusões de que a orientação para o mercado pode melhorar as capacidades de marketing na medida em que tal orientação prioriza o planejamento de marketing e programas de implementação de marketing (O'Cass et al., 2012). A capacidade das PMEs de monitorar as atividades dos concorrentes facilita o planejamento e a execução de seus programas de marketing.

A coordenação interfuncional tem uma relação significativa com as capacidades de arquitetura de marketing. Uma relação harmoniosa entre as funções facilita o compartilhamento de recursos, permitindo que as empresas planejem e implementem facilmente suas estratégias de marketing. Esses resultados estão alinhados com os achados que afirmam que o envolvimento de todas as funções internas da organização, o compartilhamento de informações e as relações harmoniosas entre as funções permitirão que as empresas atinjam seus mercados-alvo com facilidade (Song et al., 2000; Mohsen, 2013; Song, Parry, \& Ferna, 2010). Uma boa coordenação entre as funções aumenta a capacidade dos pequenos e médios empresários de planejar e colocar em prática seus programas de marketing.

A orientação para o cliente apresenta uma relação significativa com o desempenho da empresa. Atender às necessidades dos clientes estimula as empresas a inovarem em produtos que possam oferecer valor agregado e 0 melhor serviço, aumentando, assim, seus números de vendas. Aumentar os números de vendas tem o potencial de aumentar o desempenho da empresa. Isso está de acordo com os resultados de pesquisas que mostram que a orientação para o cliente tem um grande papel no apoio a melhorias no desempenho da empresa (Asomaning \& Abdulai, 2015; Feng et al., 2019; Neneh, 2018). A capacidade de entender os clientes determinará o sucesso das PMEs.

A orientação para a concorrência apresenta uma relação significativa com o desempenho da empresa. A orientação para a concorrência também facilita o planejamento e a implementação de marketing que acompanham as mudanças das necessidades do mercado-alvo, aumentando potencialmente o desempenho da empresa. Isso é apoiado pela constatação de que entender os pontos fortes e fracos dos concorrentes, conhecer suas estratégias e ações e ser capaz de responder rapidamente às suas ações têm um efeito no desempenho da empresa (Chin et al., 2013). As PMEs capazes de monitorar adequadamente as atividades dos concorrentes conseguem responder rapidamente a eles, e isso se torna um fator determinante do desempenho da empresa.

A coordenação interfuncional apresenta uma relação significativa com o desempenho da empresa. A capacidade de integrar todas as funções internas de uma organização facilita o compartilhamento de recursos de modo a criar valor agregado para o mercado-alvo. As empresas que desejam vencer a concorrência e melhorar seu próprio desempenho devem ser capazes de integrar todas as suas funções internas. Isso está de acordo com a constatação de que a coordenação interfuncional tem uma forte relação com o desempenho da empresa (Namusonge \& Nuriri, 2017; Suliyanto \& Rahab, 2012). Uma boa colaboração entre funções na organização determinará o desempenho da empresa.

As capacidades de arquitetura de marketing apresentam uma relação significativa com o desempenho da empresa. Uma empresa precisa ter uma estratégia de planejamento e implementação de marketing eficaz para atender às necessidades de seus clientes. 0 planejamento e a implementação adequados de marketing terão impacto no desempenho e no crescimento da empresa. Isso é apoiado pela constatação de que a arquitetura das capacidades organizacionais influencia o sucesso da empresa (Chikwe et al., 2018), e as capacidades de 
arquitetura de marketing possuem uma relação com o desempenho da empresa (Hamdani et al., 2018; Seifi \& Dalvi, 2014). As capacidades de arquitetura de marketing possuem um grande papel como determinantes do desempenho das PMEs.

As capacidades de arquitetura de marketing possuem um papel importante na mediação das relações entre a orientação para o mercado e as dimensões do desempenho da empresa. As empresas que compreendem as necessidades de seus clientes e como satisfazê-las conseguem responder rapidamente, criando produtos e serviços que atendam aos desejos de seus clientes. A capacidade da empresa de compreender as necessidades dos clientes e como satisfazê-las facilitará suas estratégias de planejamento e implementação de marketing, o que acabará tendo um impacto no seu desempenho. Da mesma forma, as empresas que conhecem os pontos fortes e fracos de seus concorrentes serão capazes de responder rapidamente às ações destes. A orientação para a concorrência facilita o planejamento e a implementação de marketing de acordo com as necessidades do mercado-alvo, proporcionando à empresa o potencial de melhorar seu desempenho. A capacidade da empresa de coordenar todas as funções internas tornará mais fácil compartilhar informações e recursos e colaborar na formulação de estratégias para a criação de valor agregado para os clientes. A coordenação interfuncional harmoniosa facilitará a formulação de estratégias de planejamento e implementação de marketing, afetando, assim, o desempenho da empresa.

\section{CONCLUSÃO E IMPLICAÇÕES}

Melhorar o desempenho de PMEs requer a criação de capacidades de arquitetura de marketing. Uma cultura de orientação para o mercado na forma de comportamento, incluindo-se aí a orientação para o cliente, a orientação para a concorrência e a coordenação interfuncional, será a base sobre a qual as capacidades de arquitetura de marketing podem ser desenvolvidas com o potencial de melhorar o desempenho da empresa. Espera-se que a orientação para o cliente seja capaz de compreender o mercado-alvo ao longo do tempo, a fim de criar valor para o cliente. A capacidade da empresa de entender os clientes tornará mais fácil atender às necessidades dos clientes e criar valor para eles. 0 aumento do número de clientes melhorará o desempenho da empresa. Espera-se que a orientação para concorrência obtenha informações sobre os principais e potenciais concorrentes, de modo a compreender seus pontos fortes e fracos. Os concorrentes podem ser compreendidos por meio do monitoramento de seus esforços de marketing, da criação de produtos melhores do que os deles, de respostas às mudanças em seus preços e respostas rápidas às suas atividades, o que levará à realização de uma arquitetura de marketing eficaz e eficiente, capaz de influenciar e melhorar o desempenho da empresa. A coordenação interfuncional é necessária para integrar todas as funções internas da organização a fim de facilitar a coordenação e compartilhar informações e recursos para criar valor agregado para o mercado-alvo. A coordenação interfuncional, que envolve construir o trabalho em equipe, compartilhar informações, colaborar na criação de produtos melhores do que os dos concorrentes e formular estratégias conjuntas para lidar com os concorrentes, levará à realização de uma boa arquitetura de marketing para melhorar o desempenho da empresa.

Em termos de implicações gerenciais, as PMEs que buscam melhorar seu desempenho devem compreender as necessidades dos clientes, os pontos fortes e fracos dos concorrentes e uma boa coordenação interfuncional, a fim de desenvolver estratégias de marketing adequadas. Os gestores devem entender as mudanças do mercado para criar um planejamento de arquitetura de marketing adequado ao seu mercado-alvo. Uma capacidade 
de arquitetura de marketing é necessária para que as empresas possam reorganizar seus recursos de marketing e sistemas de informação. Assim, as empresas têm a oportunidade de criar, formular e implementar estratégias de marketing de maneira adequada. Espera-se que os resultados deste estudo contribuam para o desenvolvimento do conhecimento, especialmente na área de sistemas de informação de marketing e arquitetura de marketing.

\section{LIMITAÇÕES E PESQUISAS FUTURAS}

O estudo das PMEs, que possuem recursos limitados, requer atenção especial. Uma cultura de orientação para o mercado deve ser desenvolvida para criar uma arquitetura de marketing eficaz e eficiente. A discussão sobre arquitetura de marketing nesta pesquisa ainda se concentra no planejamento e implementação de marketing. Portanto, pesquisas futuras podem incluir o gerenciamento de informações de mercado como componente da arquitetura de marketing a ser implementado em grandes empresas. Assim, será possível obter uma abordagem mais abrangente da arquitetura de marketing, e isso contribuirá para o desenvolvimento da ciência da gestão de marketing.

\section{REFERÊNCIAS}

Ajay K. K., \& Jaworski, B. J. (1993). Market orientation: Antecendent and consequances. Journal of Marketing, 57(3), 53-70. doi:10.2307/1251854

Asikhia, O. (2014). Customer orientation and firm performance among Nigerian small and medium scale businesses. International Journal of Marketing Studies, 2(1), 197-212. doi:10.5539/ijms.v2n1p197

Asomaning, R., \& Abdulai, A. (2015). An empirical evidence of the market orientation: Market performance relationship in Ghanaian small businesses. Educational Research International, 4(2), 69-86.

Avlonitis, G. J., \& Giannopoulos, A. A. (2012). Balanced market orientation: Qualitative findings on a fragile equilibrium. Managing Service Quality, 22(6), 565-579. doi: 10.1108/09604521211287543

Aziz, N. A., \& Yasin, N. M. (2014). How will market orientation and external environmnet influence the performance among SMES in the agri-food sector in Malaysia? International Business Research, 3(3), 154. doi: 10.5539/ibr.v3n3p154

Barney, J. (1991). Firm resources and sustained competitive advantage. Journal of Management, 17(1), 99-120. doi: $10.1177 / 014920639101700108$

Baron, R. M., \& Kenny, D. A. (1986). The moderator-mediator variable distinction in social psychological research: Conceptual, strategic, and statistical considerations. Journal of Personality and Social Psychology, 51(6), 1173-1182. doi: 10.1037/0022-3514.51.6.1173

Capron, L., \& Hulland, J. (1999). Redeployment of brands, sales forces, and general marketing management expertise following horizontal acquisitions: A resource-based view. Journal of Marketing, 63(2), 41-54. doi: 10.2307/1251944

Chikwe, J. E., Biriowu, S. C., \& Harcourt, P. (2018). Organizational capabilities architecture: The drivers areas sustainable hospotolity firms in South Nigeria. Ceka International Journal of Finance \& Management Sciences, 5(2), 1-11.
Chin, C. H., Lo, M. C., \& Ramayah, T. (2013). Market orientation and organizational performance: The moderat ing role of service quality. SAGE Open, 3(4), 1-14. doi: $10.1177 / 2158244013512664$

Cho, H. J., \& Pucik, V. (2005). Relationship between innovativeness, quality, growth, profitability, and market value. Strategic Management Journal, 26(6), 555-575. doi: 10.1002/smj.461

Davcik, N. S., \& Sharma, P. (2016). Marketing resources, performance, and competitive advantage: A review and future research directions. Journal of Business Research, 69(12), 55475552. doi: 10.1016/j.jbusres.2016.04.169

Day, G. S. (1994). The capabilities of market-driven organizations. Journal of Marketing, 58(4), 37-52. https:// doi: $10.1177 / 002224299405800404$

Day, G. S., \& Wensley, R. (1988). Assessing advantage: for framework diagnosing superiority competitive. Journal of Marketing, 52(2), 1-20. doi: 10.1177/002224298805200201

Dubihlela, J., \& Sandada, M. (2014). Impact of strategic planning on small and medium-sized enterprises' (SMEs) performance: The role of employee participation, implementation incentives and evaluation and control. Journal of Economics, 5(1), 45-55. doi: 10.1080/09765239.2014.11884983

Feng, T., Wang, D., Lawton, A., \& Luo, B. N. (2019, July). Customer orientation and firm performance: The joint moderating effects of ethical leadership and competitive intensity. Journal of Business Research, 100, 111-121. doi: 10.1016/j.jbusres.2019.03.021

Foumani, S. A., \& Chirani, E. (2012). Marketing knowledge management in business organizations. Arabian Journal of Business and Management Review, 1(1), 44-49. doi: $10.12816 / 0003608$

Galunic, D. C., \& Rodan, S. (1998). Resource recombinations in the firm: Knowledge structures and the potential for schumpeterian innovation. Strategic Management Journal, 19(12), 11931201. doi: 10.1002/(SICl)1097-0266(1998120)19:12〈1193::AID$\mathrm{SMJ} 533.0 . \mathrm{CO} ; 2-\mathrm{F}$ 
Gholami, S., \& Birjandi, M. (2016). The effect of market orientation and entrepreneurial orientation on the performance of SMEs. Journal of Current Research in Science, 1(5), 361-369. doi: 10.1016/j.jbusvent.2006.05.003

Hair, J. F., Black, W. C., Babin, B. J., \& Anderson, R. E. (2010). Multivariate data analysis (7th ed.). New York, USA: Prentice Hall.

Hamdani, N. A., Susanto, T., \& Maulani, G. A. F. (2018). Framework of architectural marketing capabilities in regional development bank. International Journal of Engineering \& Technology, 7(3.25), 166-169. doi: 10.14419/ijet.v7i3.25.17539

Han, J. K., Kim, N., Han, J. K., Kim, N., \& Srivastava, R. K. (1998, October). Market orientation and organizational performance: Is innovation a missing link? Journal of Marketing, 62(4), 3045. doi: $10.1177 / 002224299806200403$

Henderson, R. M., \& Clark, K. B. (1990). Architectural innovation: The reconfiguration of existing product technologies and the failure of established firms. Administrative Science Quarterly, 35(1), 1-9. doi: $10.2307 / 2393549$

Herman, H., Hady, H., \& Arafah, W. (2018). The influence of market orientation and product innovation on the competitive advantage and its implication toward small and medium enterprises (UKM) performance. International Journal of Science and Engineering Invention, 4(8), 8-21. doi: 10.23958/ ijsei/volo4-io8/02

Ho, K. L. P., Nguyen, C. N., Adhikari, R., Miles, M. P., \& Bonney, L. (2018). Exploring market orientation, innovation, and fi nancial performance in agricultural value chains in emerging economies. Journal of Innovation \& Knowledge, 3(3), 154-163. doi: 10.1016/j.jik.2017.03.008

Hwang, Y. I., \& Chung, S. (2018). Market orientation, social responsibility, and performance in Korea's healthcare industry. International Journal of Healthcare Management, 11(4), 325-332. doi: 10.1080/20479700.2017.1404728

Idrus, S. Al, Ahmar, A. S., \& Abdussakir, A. (May, 2018). Contribution of organizational learning and market orientation on business unit performance mediated by job satisfaction at dairy cattle milk cooperatives in East Java, Indonesia. Journal of Reviews on Global Economics, 7, 207-216. doi: 10.6000/19297092.2018.07.19

Javidan, M. (1998). Core competence: What does it mean in practice? Long Range Planning, 31(1), 60-71. doi: 10.1016/ s0024-6301(97)00091-5

Kanovska, L., \& Tomaskova, E. (2012). Interfunctional coordination at hi-tech firms. Enzinerine Ekonomika-Enginering Economic, 23(1), 70-76. doi: 10.5755/jo1.ee.23.1.1224

Kaswuri, A.H., Ramayah, M. A., \& Aldakhil, T. A. M.. (2017). The effect of market orientation as a mediating variable in the relationship between entrepreneurial orientation and SMEs performance. Nankai Business Review International, 8(4), 404-423. doi: 10.1108/NBRI-10-2016-0035

Kharabsheh, R., Jarrar, K., \& Simenonva, B. (2014). The impact of competitive strategies on responsive market orientation, proactive market orientation, learning orientation and organizational performance. Journal of Strategic Marketing, 23(5), 1-13. https://doi 10.1080/0965254X.2014.970217

Jaworski, B. J., \& Kohli, A. K. (1993). Market orientation: Antecendents and consequences. Journal of Marketing, 57(3), 53-70. doi:10.2307/1251854
Lee, Y. K., Kim, S. H., Seo, M. K., \& Hight, S. K. (2015, January). Market orientation and business performance: Evidence from franchising industry. International Journal of Hospitality Management, 44, 28-37. doi: 10.1016/j.ijhm.2014.09.008

Lukas, B. A., \& Ferrell, O. C. (2000). The effect of market orientation on product innovation. Journal of the Academy of Marketing Science, 28(2), 239-247. doi: 10.1177/0092070300282005

Mamun, A. Al, Mohiuddin, M., Fazal, S. A., \& Ahmad, G. B. (2018). Effect of entrepreneurial and market orientation on consumer engagement and performance of manufacturing SMEs. Management Research Review, 41(1), 133-147. doi: 10.1108/ MRR-04-2017-0102

Micheels, E. T., \& Gow, H. R. (2008). Market orientation, innovation and entrepreneurship: An empirical examination of the Illinois beef industry. International food and Agribusiness Management Review, 11(3), 1-26. doi: 10.22004/ ag.econ. 53649

Mohsen, K. (2013, September). Enhancing Inter functional coordination and marketing performance: utilization of the motivation/ability/opportunity framework. EBS Working Papers WP2013-9, University of Essex, Colchester.

Mokwa, M. P., McKee, D. O., Varadarajan, P. P., \& Conant, J. S. (1992). Success procedur and failure preventer marketing skill: A social learning theory interpretation. Journal of the Academy of Marketing Science, 20(1), 17-26. Retrieved from http://hjb. sagepub.com.proxy.lib.umich.edu/content/9/2/183.full. pdf + html

Moorman, C., \& Slotegraaf, R. J. (1999). The contingency value of complementary capabilities in product development. Journal of Marketing Research, 36(2), 239-257. doi: 10.2307/3152096

Morgan, N. A. (2012). Marketing and business performance. Journal of the Academy of Marketing Science, 40(1), 102-119. https://doi.org/10.1007/s11747-011-0279-9

Morgan, N. A., Slotegraaf, R. J., \& Vorhies, D. W. (2009). Linking marketing capabilities with profit growth. International Journal of Research in Marketing, 26(4), 284-293. https://doi. org/10.1016/j.ijresmar.2009.06.005

Narver, J. C., \& Slater, S. F. (1990). The effect of a market orientation on business profitability. Journal of Marketing. 54(4), 20-35. doi: 10.1177/002224299005400403

Neneh, B. N. (2018). Customer orientation and SME performance: The role of networking ties. African Journal of Economic and Management Studies, 9(2), 178-196. https://doi.org/10.1108/ AJEMS-03-2017-0043

Nduriri, M. W., \& Namusonge, D. M. (2017). Market orientation, entrepreneurial orientations and performance SMEs hotel in Nanyuky Town Kenya. Europenan Journal of Business and Strategic Management, 2(1), 1-14. Retrieved from https:// www.iprjb.org/journals/index.php/EJBSM/article/view/239

Nuryakin, N., Didiek A. V., \& Budi, M. (2018). Mediating effect of value creation in the relationship between relational capabilities on business performance. Contaduría y Administración, 63(1), 1-21. doi: 10.22201/ fca.24488410e.2018.1178 
O'Cass, A., Ngo, L. V., \& Siahtiri, V. (2012). Examining the marketing planning-marketing capability interface and customer-centric performance in SMEs. Journal of Strategic Marketing, 20(6), 463-481. doi: 10.1080/0965254X.2012.707675

Ogbonna, B., \& Ogwo, O. E. (2013). Market orientation and corporate performance of insurance firms in Nigeria. International Journal of Marketing Studies, 5(3), 104-116. doi: 10.5539/ijms.v5n3p104

Podsakoff, P. M., MacKenzie, S. B., \& Podsakoff, N. P. (2012). Sources of method bias in social science research and recommendations on how to control it. Annual Review of Psychology, 63(1), 539-569. doi: 10.1146/annurevpsych-120710-100452

Rahman, N. R. A., Othman, M. Z. F., Yajid, M. S. A., Rahman, S. F. A., Yaakob, A. M., Masri, R., \& Ibrahim, Z. (2018). Impact of strategic leadership on organizational performance, strategic orientation and operational strategy. Management Science Letters, 8(12), 1387-1398. doi: 10.5267/j.msl.2018.9.006

Sadikoglu, E., \& Zehir, C. (2010). Investigating the effects of innovation and employee performance on the relationship between total quality management practices and firm performance: An empirical study of Turkish firms. International Journal of Production Economics, 127(1), 13-26. doi: 10.1016/j. ijpe.2010.02.013

Seifi, R., \& Dalvi, M. R. (2014). A study of effects of specialized marketing capabilities on performance marketing unit based on Morgan et al. case: Past industry in Tehran. International Journal of Academic Research in Economics and Management Sciences, 3(1), 441-451. doi: 10.6007/ijarems/v3-i1/601

Slotegraaf, R. J., \& Dickson, P. R. (2004). The parad ox of a marketing planning capability. Journal of the Academy of Marketing Science, 32(4), 371-385. doi: 10.1177/0092070304265217

Song, M., Parry, M. E., \& Ferna, P. (2010, May). Perspective: Cross-functional integration in Spanish firms. Journal Production Innovation Management, 27, 606-615. https://doi. org/10.1111/j.1540-5885.2010.00738.x

Song, X. M., Xie, J., \& Dyer, B. (2000). Antecedents and consequences of marketing managers' conflict-handling behaviors. Journal of Marketing, 64(1), 50-65. doi: 10.1509/ jmkg.64.1.50.17989

Stam, W., Arzlanian, S., \& Elfring, T. (2014). Social capital of entrepreneurs and small firm performance: A metaanalysis of contextual and methodological moderators. Journal of Business Venturing, 29(1), 152-173. doi: 10.1016/j. jbusvent.2013.01.002
Suleiman, A. M., \& Agti, D. A. M. (2011). The impact of internal marketing on commercial banks' market orientation. International Journal of Bank Marketing, 29(4), 308-332. doi: 10.1108/02652321111145943

Suliyanto, S., \& Rahab, R. (2012). The role of market orientation and learning orientation in improving innovativeness and performance of small and medium enterprises. Asian Social Science, 8(1), 134-145. doi: 10.5539/ass.v8n1p134

Taofik, B., Halim, F., \& Ibrahim, A. (2018). The relationship between market orientation dimensions and performance of micro finance institutions. Journal of Marketing Management and Consumer Behavior, 12(3), 1-14. doi:10.24052/jbrmr/ v12iso3/art-11

Trez, G., \& Luce, F. B. (2012). Organizational structure and specialized marketing capabilities in SMEs. Marketing Intelligence and Planning, 30(2), 143-164. doi: 10.1108/02634501211211957

Udriyah, U., Tham, J., \& Azam, S. (2019). The effects of market orientation and innovation on competitive advantage and business performance of textile SMEs. Management Science Letters, 9(9), 1419-1428. doi: 10.5267/j.msl.2019.5.009

Vorhies, D. W., Harker, M., \& Rao, C. P. (1999). The capabilities and performance advantages of market-driven firms. European Journal of Marketing, 33(11/12), 1171-1202. doi: 10.1108/03090569910292339

Vorhies, D. W., \& Morgan, N. A. (2003). A configuration theory assessment of marketing organization fit with business strategy and its relationship with marketing performance. Journal of Marketing, 67(1), 100-115. doi: 10.1509/ jmkg.67.1.100.18588

Vorhies, D. W., \& Morgan, N. A. (2005). Benchmarking marketing capabilities for sustainable competitive advantage. Journal of Marketing, 69(1), 80-94. doi: 10.1509/jmkg.69.1.80.55505

Wang, C., Chen, K., \& Chen, S. (2012). Total quality management, market orientation and hotel performance: The moderating effects of external environmental factors. International Journal of Hospitality Management, 31(1), 119-129. doi: 10.1016/j. ijhm.2011.03.013

Wang, G., \& Miao, C. F. (2015). Effects of sales force market orientation on creativity, innovation implementation, and sales performance. Journal of Business Research, 68(11), 2374-2382. doi: 10.1016/j.jbusres.2015.03.041

\section{CONTRIBUIÇÃO DOS AUTORES}

Os autores declaram que participaram de todas as etapas do desenvolvimento do manuscrito. Mulyana Mulyana trabalhou na conceitualização e abordagem teórico-metodológica. A revisão teórica foi realizada por Zainuddin Zakaria. A coleta de dados foi coordenada por Rosman Mahmood. A análise de dados foi realizada por Mulyana Mulyana, Zainuddin Zakaria e Rosman Mahmood. Todos os autores trabalharam juntos na redação e revisão final do manuscrito. 